\section{(2) OPEN ACCESS}

\title{
SARS-CoV-2 vaccination responses in untreated, conventionally treated and anticytokine-treated patients with immune-mediated inflammatory diseases
}

\author{
David Simon (D) , 1,2 Koray Tascilar (D) , ${ }^{1,2}$ Filippo Fagni (D) , ${ }^{1,2}$ Gerhard Krönke, ${ }^{1,2}$ \\ Arnd Kleyer, ${ }^{1,2}$ Christine Meder, ${ }^{2,3}$ Raja Atreya, ${ }^{2,4}$ Moritz Leppkes, ${ }^{2,4}$ \\ Andreas E Kremer (D) , ${ }^{2,4}$ Andreas Ramming (D) , 1,2 Milena L Pachowsky, ${ }_{1}^{1,2}$ \\ Florian Schuch, ${ }^{5}$ Monika Ronneberger, ${ }^{5}$ Stefan Kleinert, ${ }^{5}$ Axel J Hueber, ${ }^{1,6}$ \\ Karin Manger, ${ }^{7}$ Bernhard Manger (D) , 1,2 Carola Berking, ${ }^{2,3}$ Michael Sticherling, ${ }^{2,3}$ \\ Markus F Neurath, ${ }^{2}$ Georg Schett (1) 1,2
}

\begin{abstract}
Handling editor Josef $S$ Smolen

- Additional supplemental material is published online only. To view, please visit the journal online (http://dx.doi. org/10.1136/annrheumdis2021-220461).
\end{abstract}

For numbered affiliations see end of article.

\section{Correspondence to} Professor Georg Schett, Department of Internal Medicine 3, Friedrich-Alexander University Erlangen-Nuremberg, Erlangen 91054, Germany;

georg.schett@uk-erlangen.de

DS and KT contributed equally.

Received 29 March 2021 Revised 23 April 2021 Accepted 27 April 2021 Published Online First 6 May 2021

\section{ABSTRACT}

Objectives To better understand the factors that influence the humoral immune response to vaccination against SARS-CoV-2 in patients with immune-mediated inflammatory diseases (IMIDs).

Methods Patients and controls from a large COVID-19 study, with (1) no previous history of COVID-19, (2) negative baseline anti-SARS-CoV-2 IgG test and (3) SARS-CoV-2 vaccination at least 10 days before serum collection were measured for anti-SARS-CoV-2 lgG. Demographic, disease-specific and vaccination-specific data were recorded.

Results Vaccination responses from 84 patients with IMID and 182 controls were analysed. While all controls developed anti-SARS-CoV-2 lgG, five patients with IMID failed to develop a response $(p=0.003)$. Moreover, $99.5 \%$ of controls but only $90.5 \%$ of patients with IMID developed neutralising antibody activity $(p=0.0008)$. Overall responses were delayed and reduced in patients (mean (SD): 6.47 (3.14)) compared with controls (9.36 (1.85); $p<0.001)$. Estimated marginal means $(95 \% \mathrm{Cl})$ adjusted for age, sex and time from first vaccination to sampling were 8.48 (8.12-8.85) for controls and 6.90 (6.45-7.35) for IMIDs. Significantly reduced vaccination responses pertained to untreated, conventionally and anticytokine treated patients with IMID.

Conclusions Immune responses against the SARSCoV-2 are delayed and reduced in patients with IMID. This effect is based on the disease itself rather than concomitant treatment.

\section{INTRODUCTION}

COVID-19 has developed into one of the most impactful pandemics. ${ }^{1}$ Within short times, tremendous research efforts have led to the development of effective vaccines. ${ }^{23}$ Their efficacy and safety in the general population is substantiated by a growing number of studies that demonstrate the development of protective immunity and the appearance of specific antibodies against SARS-CoV-2. ${ }^{5}$

The development of protective immunity requires a functional immune system, which can be impaired

\section{Key messages}

What is already known about this subject?

- While it is known that SARS-CoV-2 vaccination is effective in the general population, virtually no data on the efficacy and safety of the vaccine in patients with immune-mediated inflammatory diseases exist at the moment. Most importantly, it is not known whether the disease itself or the respective immunemodulatory therapy may affect the immune response to the SARS-CoV-2 vaccine.

What does this study add?

- The study shows that one out of 10 patients with an immune-mediated inflammatory disease fails to develop neutralizing antibodies after SARS-CoV-2 vaccination, while it is only 1 out of 100 in healthy controls.

- Decreased immune response to SARS-CoV-2 vaccination is immanent to the presence of an immune-mediated inflammatory disease but not related to the individual immune-modulatory treatments.

How might this impact on clinical practice or future developments?

- These data suggest that humoral immune responses to SARS-CoV-2 vaccination need to be assessed in patients with immune-mediated inflammatory diseases in order to ascertain protective immunity.

by diseases or treatments. Patients affected by immune-mediated inflammatory diseases (IMIDs) show aberrant immune responses, increased risk to infections and are exposed by drugs that interfere with immune pathways. Hence, responses of patients with IMID to immunisation against SARS-CoV-2 may be altered. Furthermore, IMIDs are usually associated with comorbidities that increase the risk for severe courses of COVID- $19 .{ }^{6}$ 
In accordance, the risk for severe courses of COVID-19 has reported to be higher in patients with IMID. ${ }^{7}$ For this reason, it seems reasonable to give patients with IMID preferential access to vaccination. ${ }^{89}$

At present, however, there is a tremendous paucity of data, that could guide physicians in how individual IMIDs and immunemodulatory treatments associated with these IMIDs would influence the immune response to SARS-CoV-2 vaccination. Patients with IMIDs and those receiving immune-modulatory treatments were excluded from the phase III vaccine trials. One recent small study suggested that vaccination against SARS-CoV-2 works in patients with IMIDs, but leaves it open whether and how the presence of the disease or the use of specific drugs influences the immune responses. ${ }^{10}$ We therefore analysed the first vaccination responses in large longitudinal COVID-19 study that follows antibody responses to SARS-CoV-2 in healthy individuals and patients with IMID over time. ${ }^{11}$

\section{METHODS}

\section{Participants}

Patients with IMID and healthy controls were recruited from a large longitudinal COVID-19 study at the Deutsche Zentrum fuer Immuntherapie that has been initiated in February 2020 and monitors anti-SARS-CoV-2 antibody responses as well as respiratory infections including COVID-19 in healthy controls and patients with IMID. ${ }^{11}$ All patients and controls with (1) no previous history of COVID-19, (2) negative anti-SARS-CoV-2 IgG test in December 2020/January 2021 and (3) having received at least one shot of the BNT162b2 mRNA SARS-CoV-2 vaccine (BioNTech/Pfizer) more than 10 days before serum collection were included into this study. Demographic (age, sex, body mass index, comorbidities), disease-specific (type of IMID, type of treatment) and vaccination (date, type of vaccine, adverse reactions) data were recorded.

IgG antibodies against the $\mathrm{S} 1$ domain of the spike protein of SARS-CoV-2 were tested by the recent CE version (April 2020) of the commercial ELISA from Euroimmun (Lübeck, Germany) using the EUROIMMUN Analyzer I platform and according to the manufacturers protocol. All analyses were done in duplicates. Optical density (OD) was determined at $450 \mathrm{~nm}$ with reference wavelength at $630 \mathrm{~nm}$. A cut-off of $\geq 0.8$ (OD $450 \mathrm{~nm}$ ) was considered as positive. To assess neutralisation activity of the antibodies, a CE- In Vitro Diagnostics (CE-IVD)-certified SARS-CoV-2 surrogate virus neutralisation assay (cPASS, Medac, Wedel, Germany) was used. This assay measures the potential of antibodies to inhibit the binding of a labelled SARS-CoV-2 receptor-binding domain (RBD) to coated angiotensin-converting enzyme-2 (ACE2. A cut-off of 30\% inhibition was considered as positive, according to the manufacture's instructions.

\section{Statistical analysis}

We described participant characteristics using appropriate summary statistics for continuous and categorical data. Antibody levels over time were visually analysed using scatter plot smoothers based on generalised additive models to explore the course of response after the initial vaccine dose. To explore the association of vaccination response with demographic characteristics and disease status, we fitted linear regression models with the OD values from the antibody assay as the dependent variable and participant/treatment groups, age, sex and time after the first vaccine dose as independent variables. Since a non-linear relationship between time and vaccine response is expected, we included days after first vaccination in the model using restricted cubic splines with three knots that were placed based on the inflection points on the scatterplot smoother. This provided a better fit compared with linear or quadratic terms for time.

We reported empirical group means for description. For between-group comparisons, we used estimated marginal means (ie, least square means) from the model. These were weighted for imbalances between covariate categories, averaged over sex and were conditional on overall mean age and mean duration after vaccination in order to account for differences between healthy controls and IMID treatment groups. T tests were used for comparisons. We used Fisher's exact test to compare categorical vaccine response between groups. Two-sided unadjusted $\mathrm{p}$ values $<0.05$ were considered significant. All analyses were carried out using the open-source R software V.4.0.1 (R Foundation for Statistical Computing, Vienna, Austria) running under the GUI RStudio (RStudio corp, Boston, Massachusetts, USA) with the 'rms' and 'emmeans' packages.

\section{RESULTS}

\section{Characteristics of patients and controls}

From 28 December 2020 until 20 March 2021, 84 patients with IMID (mean age $53.1 \pm 17.0$ years, $65.5 \%$ females) and 182 healthy controls $(40.8 \pm 12.0$ years, $57.1 \%$ females $)$ had received at least one shot of the SARS-CoV-2 vaccine (Biontec/ Pfizer) at least 10 days ago. The vast majority (96\%) of subjects had received two shots of the vaccination. All of these individuals did not have a history of COVID-19 in 2020 and were antibody negative before the vaccination (testing in December 2020). Most patients with IMID had spondyloarthritis (SpA/ psoriatic arthritis) (32.1\%), followed by rheumatoid arthritis (RA) (29.8\%), inflammatory bowel disease (9.5\%), psoriasis $(9.5 \%)$ and systemic IMIDs (table 1 ). About $42.9 \%$ of the patients received biologic (b) or targeted synthetic (ts) diseasemodifying antirheumatic drugs (DMARDs), $23.9 \%$ were treated conventional synthetic (cs) DMARDs, while $28.6 \%$ received no treatment.

\section{Dynamics of SARS-CoV-2 vaccination responses in patients with IMID and controls}

All controls responded to the vaccine, reaching positive (OD $>0.8$ ) anti-SARS-CoV-2 IgG antibodies. Positive antibody responses in controls were observed as early as 11 days after the first vaccination. Most patients with IMID also developed positive anti-SARS-CoV-2 IgG antibodies. However, vaccination failed in five patients with IMID ( $\mathrm{p}=0.003$; Fisher's exact test). In three of them, lack of immunogenicity was even detectable 11, 27 and 39 days after the second vaccination (one without therapy, one patient with RA treated with baricitinib and one patient with SpA with secukinumab). Patients with IMID showed relatively large OD difference shortly after the second vaccination compared with controls, but this difference converged over time (figure 1A). In a linear model implementing group-time interactions, the adjusted mean difference between controls and patients with IMID at day 28 after the first vaccine administration was 2.21 (95\% CI: 1.28 to $3.13, \mathrm{p}<0.001$ ) reducing to 0.07 (95\% CI: -1.27 to $1.12, \mathrm{p}=0.90$ ) at day 70 .

Assessment of neutralisation activity of anti-SARS-CoV-2 antibodies, using an assay that measures their potential to block binding of RBD to ACE2 showed that 99.5\% (181/182) of controls developed neutralising antibodies, while only $90.5 \%$ (76/84) of patients with IMID developed neutralising activity $(p=0.0008$; Fisher's exact test). Among those failing to develop 
Table 1 Demographics and clinical characteristics of patients with IMID and controls

\begin{tabular}{|c|c|c|}
\hline & IMIDs & HC \\
\hline $\mathrm{N}$ & 84 & 182 \\
\hline \multicolumn{3}{|l|}{ Demographic characteristics } \\
\hline Age, years & $53.1 \pm 17.0$ & $40.8 \pm 12.0$ \\
\hline Females, N (\%) & $55(65.5)$ & $104(57.1)$ \\
\hline BMI & $26.8 \pm 5.8$ & $24.7 \pm 4.1$ \\
\hline Current smokers, $\mathrm{N}(\%)$ & $14(16.7)$ & $31(17.0)$ \\
\hline \multicolumn{3}{|l|}{ Comorbidities } \\
\hline Diabetes & $6(7.1)$ & $2(1.1)$ \\
\hline Hypertension & $21(25.0)$ & $19(10.4)$ \\
\hline History of CV event & $1(1.2)$ & $1(1.0)$ \\
\hline History of thrombotic event & 0 & 0 \\
\hline \multicolumn{3}{|l|}{ Type of IMID } \\
\hline SpA, N (\%) & $27(32.1)$ & 0 \\
\hline RA, N (\%) & $25(29.8)$ & 0 \\
\hline IBD, N (\%) & $8(9.5)$ & 0 \\
\hline Psoriasis, N (\%) & $8(9.5)$ & 0 \\
\hline Systemic ${ }^{*}, N(\%)$ & $16(19.1)$ & 0 \\
\hline \multicolumn{3}{|l|}{ Immune-modulatory therapy } \\
\hline No treatment, $\mathrm{N}(\%)$ & $24(28.6)$ & 0 \\
\hline Glucocorticoids, N (\%) & $10(11.9)$ & 0 \\
\hline csDMARDs monotherapy, $\mathrm{N}(\%)$ & $20(23.9)$ & 0 \\
\hline MTX, N (\%) & $16(19.1)$ & 0 \\
\hline Hydroxychloroquine, N (\%) & $3(3.6)$ & 0 \\
\hline Sulfasalazine, N (\%) & $1(1.2)$ & 0 \\
\hline bDMARDs/tsDMARDs, N (\%) & $36(42.9)$ & 0 \\
\hline TNF inhibitors, N (\%) & $11(13.1)$ & 0 \\
\hline IL-6 inhibitors, N (\%) & $3(3.6)$ & 0 \\
\hline IL-23 inhibitors, N (\%) & $6(7.1)$ & 0 \\
\hline IL-17 inhibitors, N (\%) & $7(8.3)$ & 0 \\
\hline JAK inhibitors, N (\%) & $6(7.1)$ & 0 \\
\hline Otherst, N (\%) & $3(3.6)$ & 0 \\
\hline
\end{tabular}

*Systemic lupus erythematosus, systemic sclerosis, IgG4-related disease, periodic fever syndromes, giant cell arteriitis, granulomatosis with polyangiitis and polymyalgia rheumatic.

†Apremilast, canakinumab and vedolizumab.

bDMARDs, biological disease-modifying antirheumatic drugs; BMI, body mass index; csDMARDs, conventional synthetic disease-modifying antirheumatic drugs; $\mathrm{CV}$, cardiovascular; HC, healthy controls; IBD, inflammatory bowel disease; IL, interleukin; IMIDs, immune-mediated inflammatory diseases; JAK, Janus kinase; MTX, methotrexate; RA, rheumatoid arthritis; SpA, spondyloarthritis (including axial spondyloarthritis and psoriatic arthritis); TNF, tumour necrosis factor; tsDMARDs, targeted-synthetic disease-modifying antirheumatic drugs.

neutralising activity were three Janus kinase inhibitors, two methotrexate, one interleukin-17 inhibitor treated and two untreated patients with IMID .

\section{Comparison of SARS-CoV-2 vaccination responses in patients with IMID and controls}

Overall mean (SD) OD values were 6.47 (3.14) in patients with IMID compared with $9.36(1.85)$ in controls (adjusted mean difference $1.58,95 \% \mathrm{CI}: 0.98$ to $2.19, \mathrm{p}<0.001)$. Estimated marginal means $(95 \% \mathrm{CI})$ adjusted for age, sex and time elapsed from first vaccination to sampling date were 8.48 (8.12 to 8.85) for controls and 6.90 (6.45 to 7.35) for IMIDs (table 2). Linear regression model showed that vaccine responses were influenced by the presence of IMID, age, sex and time elapsed from vaccination (online supplemental table 3 ).
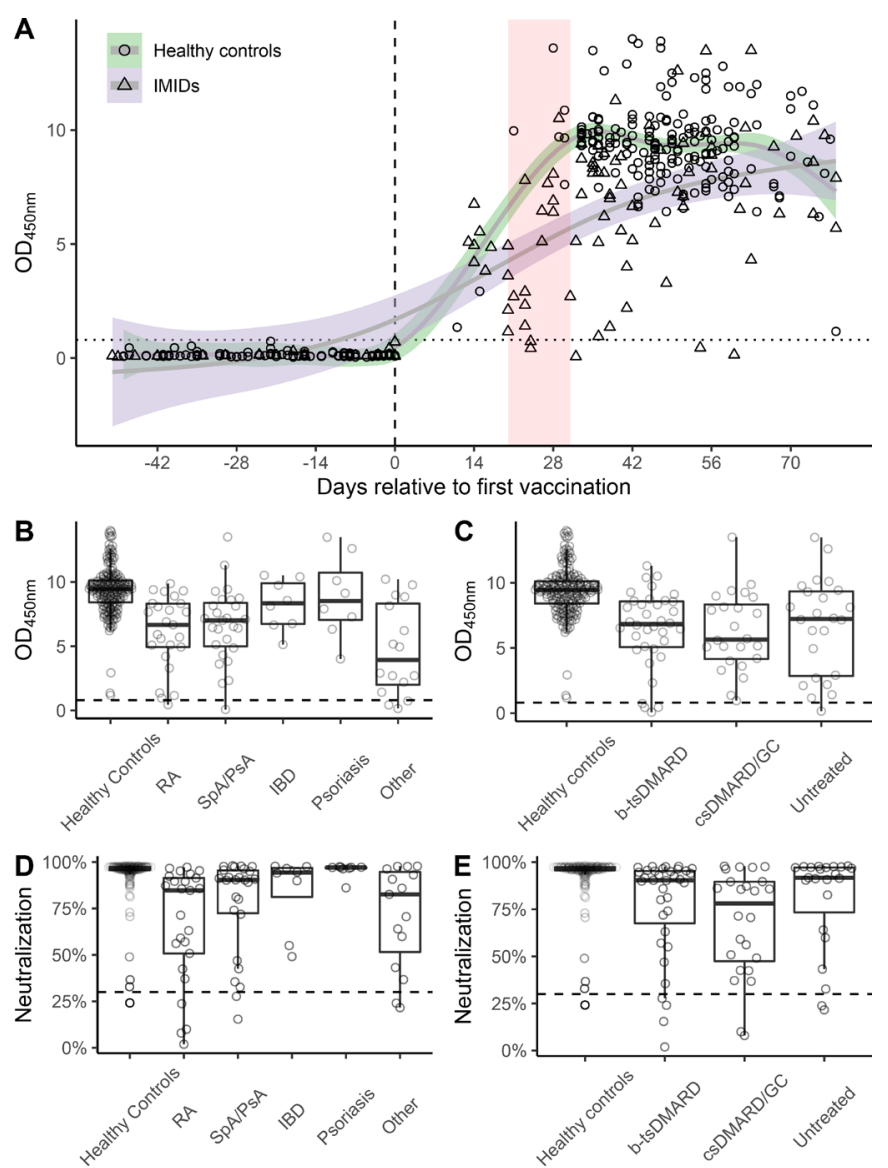

Figure 1 Temporal pattern of vaccination response and antibody levels in different disease and treatment groups. (A) Temporal course of anti-SARS-CoV-2 antibody formation after first and second mRNA vaccine doses, first vaccination is depicted by a dotted vertical line, second vaccination by a red vertical band, smoothed plots show time-conditional mean antibody levels in healthy controls and IMID subgroups. (B) Distribution of antibody levels by type of treatment (B) and diagnosis (C). Dotted horizontal lines represent OD cut-off of $\geq 0.8$ (OD $450 \mathrm{~nm})$. (D, E) Distribution of neutralisation activity of the antibodies based on per cent inhibition of binding of the receptorbinding domain to angiotensin-converting enzyme-2 by type of treatment (D) and diagnosis (E). Dotted horizontal lines represent cut-off of $\geq 30 \%$ inhibition. bDMARDs, biological disease-modifying antirheumatic drugs; csDMARDs, conventional synthetic diseasemodifying antirheumatic drugs; IBD, inflammatory bowel disease; $O D$, optical density; PsA, psoriatic arthritis; RA, rheumatoid arthritis; SpA, spondyloarthritis; tsDMARDs, targeted-synthetic disease-modifying antirheumatic drugs.

Table 2 Empirical and estimated marginal means by study groups and treatment

\begin{tabular}{lll}
\hline Group & Empirical mean (SD) & EMM $^{*}$ (95\% Cl) \\
\hline Controls & $9.36(1.85)$ & $8.48(8.12$ to 8.85$)$ \\
IMIDs all & $6.47(3.14)$ & $6.90(6.45$ to 7.35$)$ \\
IMIDs b/tsDMARDs & $6.49(2.91)$ & $6.90(6.22$ to 7.58$)$ \\
csDMARDs & $6.26(3.00)$ & $6.67(5.84$ to 7.50$)$ \\
Untreated & $6.64(3.70)$ & $7.13(6.30$ to 7.96$)$ \\
\hline
\end{tabular}

${ }^{*}$ Adjusted for age, sex, time elapsed from first vaccination date to sampling date. bDMARDs, biological disease-modifying antirheumatic drugs; csDMARDs, conventional synthetic disease-modifying antirheumatic drugs; EMM, estimated marginal mean; IMIDs, immune-mediated inflammatory diseases; tsDMARDs, targeted-synthetic disease-modifying antirheumatic drugs. 


\section{Effect of IMID group and immune-modulatory treatment on vaccination responses}

When analysing immune response to SARS-CoV-2 vaccination in different IMID groups, overall mean ODs were similar across IMIDs and lower than that of controls. We did not detect any significant difference between diseases based on adjusted mean differences (figure 1B). When analysing different treatment regimen (no treatment, csDMARDs, bDMARDs/tsDMARDs), we found that patients with IMID treated with bDMARDs/ tsDMARDs did not show a different response compared with patients receiving csDMARDs (6.49 (2.91) vs 6.26 (3.00); mean diff. $95 \%$ CI $0.23(-0.83$ to 1.30$), \mathrm{p}=0.97)$ or to those without treatment $(6.49(2.91)$ vs $6.64(3.70) ;-0.22(-1.28$ to 0.83$)$, $\mathrm{p}=0.97$ ) (online supplemental table 2 ). In contrast, all three IMID treatment groups showed lower OD values than controls (online supplemental table 2; figure 1C). Responses in individual bDMARDs treatments are depicted in online supplemental table 3.

\section{Tolerability of SARS-CoV-2 vaccination in patients with IMID}

Side effects of vaccination were assessed in 70 patients with IMID and 164 controls. Side effects were generally more frequent after the second vaccination. Injection side pain was most frequently observed in both groups. Many side effects (injection side reaction, headache, chills, arthralgia) were less frequent in patients and in controls (online supplemental table 4).

\section{DISCUSSION}

This study shows that SARS-CoV-2 vaccination essentially works in patients with IMID but responses are delayed and reduced. A minority of patients with IMID did not respond to the vaccine even after second immunisation, suggesting that in some cases the measurement of antibody levels after vaccination might be useful to ascertain development of immunity. In accordance, only $0.5 \%$ of the controls failed to develop neutralising antibody activity, while such failures were observed in $9.5 \%$ of the patients with IMID. Thus, roughly 1 out of 10 patients with IMID fails to develop neutralising antibodies after SARS-CoV-2 vaccination, while it is only 1 out of 100 in the controls. This findings contrast the data from a small group of 26 patients with IMID suggesting that all patients with IMID respond to the vaccine. ${ }^{10}$

Delayed antibody responses to the SARS-CoV-2 vaccine may suggest an effect of immune-modulatory treatments. However, we could not objectify this hypothesis, as also patients with IMID without treatment had lower antibody responses than controls and furthermore no differences between csDMARDs and $b / t s D M A R D$ treated patients were found. Of note this IMID cohort did not comprise rituximab-treated patients, in whom antibody responses are abrogated. ${ }^{12}$ Hence, delayed antibody responses seem to be a disease rather than a treatment-related effect. In addition to the presence or absence of IMIDs, sex and age affected SARS-CoV-2 vaccination responses, which is in accordance with published work. ${ }^{13} 14$

In conclusion, our study provides evidence that while vaccination against SARS-CoV-2 is well-tolerated and even associated with lower incidence of side effects in patients with IMID, its efficacy is somewhat delayed and reduced. Nonetheless, the data also show that, in principle, patients with IMID respond to SARS-CoV-2 vaccination, supporting an aggressive vaccination strategy. In addition, cell-mediated responses to vaccination, which were not analysed in this study, may additionally contribute to anti-SARS-CoV-2 immunity in patients with IMID.$^{15}$

\section{Author affiliations}

'Department of Internal Medicine 3 - Rheumatology and Immunology, FriedrichAlexander University Erlangen-Nuremberg and Universitätsklinikum Erlangen,

Erlangen, Germany

${ }^{2}$ Deutsches Zentrum fuer Immuntherapie (DZI), Friedrich-Alexander University Erlangen-Nuremberg and Universitätsklinikum Erlangen, Erlangen, Germany ${ }^{3}$ Department of Dermatology, Friedrich-Alexander University Erlangen-Nuremberg, Erlangen, Germany

${ }^{4}$ Department of Internal Medicine 1, Friedrich-Alexander University Erlangen-

Nuremberg, Erlangen, Germany

${ }^{5}$ Rheumatology Practice, Erlangen, Germany

${ }^{6}$ Section Rheumatology, Sozialstiftung Bamberg, Bamberg, Germany

${ }^{7}$ Rheumatology Practice Bamberg, Erlangen, Germany

Twitter Koray Tascilar @KorayTascilar

Contributors DS, KT, AK, GK, MN and GS were involved in study design. Sample collection was done by DS, GK, RA, FS, MS, SF, AJH, KM, BM, CB, MS, AK, MN and GS. Experiments and data analysis were performed by DS, KT, FF and KT. DS and KT were responsible for tables and figure. Data interpretation was done by $D S, K T, C B$, $M S, A K, M N$ and GS. Writing of the manuscript was done by DS, KT, FF, MN and GS. All authors were involved in critical proof reading of the manuscript.

Funding The study was supported by the Deutsche Forschungsgemeinschaft (DFG-FOR2886 PANDORA and the CRC1181 Checkpoints for Resolution of Inflammation). Additional funding was received by the Bundesministerium für Bildung und Forschung (BMBF; project MASCARA), the ERC Synergy grant 4D Nanoscope, the IMI funded project RTCure, the Emerging Fields Initiative MIRACLE of the Friedrich-Alexander-Universität Erlangen-Nürnberg, the Schreiber Stiftung and the Else Kröner-Memorial Scholarship (DS, no. 2019_EKMS.27). The present work was performed in (partial) fulfilment of the requirements for obtaining the degree 'Dr rer. biol. hum'. for DS at the Friedrich-Alexander-University ErlangenNürnberg (FAU)

Competing interests None declared.

\section{Patient consent for publication Not required.}

Ethics approval Ethical approval (\#157_20 B) to conduct this study was granted by the Institutional Review Board of the University Hospital Erlangen. Written informed consent was obtained from the study participants.

Provenance and peer review Not commissioned; externally peer reviewed.

Data availability statement All data relevant to the study are included in the article or uploaded as online supplemental information. Data are under embargo by local authorities if not included into the manuscript.

Supplemental material This content has been supplied by the author(s). It has not been vetted by BMJ Publishing Group Limited (BMJ) and may not have been peer-reviewed. Any opinions or recommendations discussed are solely those of the author(s) and are not endorsed by BMJ. BMJ disclaims all liability and responsibility arising from any reliance placed on the content. Where the content includes any translated material, BMJ does not warrant the accuracy and reliability of the translations (including but not limited to local regulations, clinical guidelines, terminology, drug names and drug dosages), and is not responsible for any error and/or omissions arising from translation and adaptation or otherwise.

Open access This is an open access article distributed in accordance with the Creative Commons Attribution Non Commercial (CC BY-NC 4.0) license, which permits others to distribute, remix, adapt, build upon this work non-commercially, and license their derivative works on different terms, provided the original work is properly cited, appropriate credit is given, any changes made indicated, and the use is non-commercial. See: http://creativecommons.org/licenses/by-nc/4.0/.

\section{ORCID iDs}

David Simon http://orcid.org/0000-0001-8310-7820

Koray Tascilar http://orcid.org/0000-0002-8109-826X

Filippo Fagni http://orcid.org/0000-0002-6122-0774

Andreas E Kremer http://orcid.org/0000-0002-9263-948X

Andreas Ramming http://orcid.org/0000-0002-7003-501X

Bernhard Manger http://orcid.org/0000-0003-2375-0069

Georg Schett http://orcid.org/0000-0001-8740-9615

\section{REFERENCES}

1 Jordan RE, Adab P, Cheng KK. Covid-19: risk factors for severe disease and death. BMJ 2020;368:m1198.

2 Polack FP, Thomas SJ, Kitchin N, et al. Safety and efficacy of the BNT162b2 mRNA Covid-19 vaccine. N Engl J Med 2020;383:2603-15. 


\section{Epidemiology}

3 Baden LR, El Sahly HM, Essink B, et al. Efficacy and safety of the mRNA-1273 SARSCoV-2 vaccine. N Engl J Med 2021;384:403-16.

4 Anderson EJ, Rouphael NG, Widge AT, et al. Safety and immunogenicity of SARSCoV-2 mRNA-1273 vaccine in older adults. N Engl J Med 2020;383:2427-38.

5 Sahin U, Muik A, Derhovanessian E, et al. COVID-19 vaccine BNT162b1 elicits human antibody and T $1 \mathrm{~T}$ cell responses. Nature 2020;586:594-9.

6 Dougados M, Soubrier M, Antunez A, et al. Prevalence of comorbidities in rheumatoid arthritis and evaluation of their monitoring: results of an international, cross-sectional study (COMORA). Ann Rheum Dis 2014;73:62-8.

7 Williamson EJ, Walker AJ, Bhaskaran K, et al. Factors associated with COVID-19related death using OpenSAFELY. Nature 2020;584:430-6.

8 Curtis JR, Johnson SR, Anthony DD, et al. American College of Rheumatology Guidance for COVID-19 Vaccination in Patients with Rheumatic and Musculoskeletal Diseases - Version 1. Arthritis Rheumatol 2021. doi:10.1002/art.41734. [Epub ahead of print: 17 Mar 2021].

9 Rondaan C, Furer V, Heijstek MW, et al. Efficacy, immunogenicity and safety of vaccination in adult patients with autoimmune inflammatory rheumatic diseases: a systematic literature review for the 2019 update of EULAR recommendations. RMD Open 2019;5:e001035

10 Geisen UM, Berner DK, Tran F. Immunogenicity and safety of anti-SARSCoV-2 mRNA vaccines in patients with chronic inflammatory conditions and immunosuppressive therapy in a monocentric cohort. Ann Rheum Dis 2021;80:1302-7.

11 Simon D, Tascilar K, Krönke G, et al. Patients with immune-mediated inflammatory diseases receiving cytokine inhibitors have low prevalence of SARS-CoV-2 seroconversion. Nat Commun 2020;11:3774.

12 D'Silva KM, Serling-Boyd N, Hsu TY-T, et al. SARS-CoV-2 antibody response after COVID-19 in patients with rheumatic disease. Ann Rheum Dis 2021;80:817-9.

13 Sakiani S, Olsen NJ, Kovacs WJ. Gonadal steroids and humoral immunity. Nat Rev Endocrinol 2013;9:56-62.

14 Scully EP, Haverfield J, Ursin RL, et al. Considering how biological sex impacts immune responses and COVID-19 outcomes. Nat Rev Immunol 2020;20:442-7.

15 Le Bert N, Tan AT, Kunasegaran K, et al. SARS-CoV-2-specific T cell immunity in cases of COVID-19 and SARS, and uninfected controls. Nature 2020;584:457-62. 